\title{
Testing uniformity for the case of a planar unknown support
}

\author{
José R. BERRENDERO ${ }^{1}$, Antonio CUEVAS ${ }^{1}$ and Beatriz PATEIRO-LÓPEZ ${ }^{2 *}$ \\ ${ }^{1}$ Universidad Autónoma de Madrid, Spain \\ ${ }^{2}$ Universidad de Santiago de Compostela, Spain
}

Key words and phrases: Convexity; distance to boundary; $\lambda$-convexity ( $r$-convexity); set estimation; uniformity test.

MSC 2010: Primary 62G10; secondary 62G05, 62G20

\begin{abstract}
A new test is proposed for the hypothesis of uniformity on bi-dimensional supports. The procedure is an adaptation of the "distance to boundary test" (DB test) proposed in Berrendero, Cuevas, \& Vázquez-Grande (2006). This new version of the DB test, called DBU test, allows us (as a novel, interesting feature) to deal with the case where the support $S$ of the underlying distribution is unknown. This means that $S$ is not specified in the null hypothesis so that, in fact, we test the null hypothesis that the underlying distribution is uniform on some support $S$ belonging to a given class $\mathcal{C}$. We pay special attention to the case that $\mathcal{C}$ is either the class of compact convex supports or the (broader) class of compact $\lambda$-convex supports (also called $r$-convex or $\alpha$-convex in the literature). The basic idea is to apply the DB test in a sort of plug-in version, where the support $S$ is approximated by using methods of set estimation. The DBU method is analyzed from both the theoretical and practical point of view, via some asymptotic results and a simulation study, respectively. The Canadian Journal of Statistics xx: 1-25; 20?? (c) 20?? Statistical Society of Canada
\end{abstract}

Résumé: Insérer votre résumé ici. We will supply a French abstract for those authors who can't prepare it themselves. La revue canadienne de statistique xx: 1-25; 20?? (C) 20?? Société statistique du Canada

\section{INTRODUCTION}

We are concerned with the problem of testing the null hypothesis

$$
H_{0} \text { : the random variable } X \text { has a uniform distribution on some support } S \text {. }
$$

We assume throughout that the available information is given by an iid sample $X_{1}, \ldots, X_{n}$ drawn from the $d$-dimensional random variable $X$.

The vast majority of theoretical developments and applications for this problem deal with either the univariate case $d=1$ or the bivariate models with $d=2$. The motivations for both situations are quite different. While the univariate uniformity tests are often motivated by the need of having good "random number generators", the bivariate uniformity problems arise usually in the setting of spatial statistics. Anyway, the bivariate problem is considerably harder in several senses. A first obvious difficulty for $d=2$ is the lack of a distribution-free procedure (such as the univariate Kolmogorov-Smirnov test) based on the empirical distribution. Also, the choice of the support is not an issue in most univariate uniformity problems, as they are naturally set out in a known interval $S=[a, b]$ which can be reduced to the standard case $[a, b]=[0,1]$; on the contrary, when we are dealing with bivariate data there is no good reason for restricting us to a

\footnotetext{
* Author to whom correspondence may be addressed.

E-mail: beatriz.pateiro@usc.es
}

(c) 20?? Statistical Society of Canada / Société statistique du Canada 
fixed support as, for example, $S=[0,1]^{2}$. Of course, $S=[0,1]^{2}$ is a relevant case, but there are many other conceivable interesting supports (such as polygons, ellipses, etc.) and one might even consider the case where $S$ is not known in advance and only a generic regularity assumption on its structure is imposed. In other words, the class $\mathcal{U}\left(\mathbb{R}^{2}\right)$ of uniform distributions with connected support in $\mathbb{R}^{2}$ is much more complicated than its one-dimensional analog, $\mathcal{U}(\mathbb{R})$. The latter is a parametric family so that, even if the support $S$ were unknown, its estimation is a simple matter based on standard methods. This is not at all the case with $\mathcal{U}\left(\mathbb{R}^{2}\right)$. Thus, it is clear that the goodness-of-fit problem to the non-parametric family $\mathcal{U}\left(\mathbb{R}^{2}\right)$ (or to appropriate sub-families of it) involves non-trivial geometric and statistical issues which lead us to the main point of this work.

\section{The purpose of this paper}

We specifically aim at developing a new uniformity test, based on an iid sample of size $n$, for the null hypothesis

$$
H_{0} \text { : the random variable } X \text { has a uniform distribution belonging to the class } \mathcal{U}_{\mathcal{C}},
$$

where $\mathcal{U}_{\mathcal{C}}$ is the class of bivariate uniform distributions whose support $S$ belongs to a given class $\mathcal{C}$ of compact supports in $\mathbb{R}^{2}$. As we will see, the natural assumption of connectedness for $S$ can be incorporated to our approach but it is not strictly needed.

Our test will consist of an adaptation of the Distance-to-Boundary Method (DB) which was proposed by Berrendero, Cuevas, \& Vázquez-Grande (2006) for the simplest, usual case that the support $S$ is completely known and specified in the null hypothesis; in the notation (1), this would amount to take a class $\mathcal{C}=\{S\}$ with a unique member. The DB method was based on calculating the distances $Y_{i}$ from the sampling observations $X_{i}$ to the boundary of the support $S$. The test checks the fit of the empirical distribution of this variables to that corresponding to the case where $H_{0}$ is true (a more detailed account will be given below). The purpose of this paper is to show that this method can be adapted to the case where the support $S$ is unknown so that we deal in fact with a general problem of type (1). Our extension of the DB procedure, which we will denote DBU test, relies on methods of set estimation (see Cuevas \& Fraiman (2009) for a survey of this topic). The basic idea is a sort of plug-in device: we apply the DB test presented in Berrendero, Cuevas, \& Vázquez-Grande (2006) replacing the support $S$ by a suitable estimator $S_{n}$. If the estimated boundary $\partial S_{n}$ approaches fast enough to the population counterpart $\partial S$, the respective critical regions in both tests (with the tests statistics calculated from $S$ and $S_{n}$, respectively) will be asymptotically equivalent.

There are many possible different choices for $\mathcal{U}_{\mathcal{C}}$ in $(1)$. We will pay especial attention to the cases where $\mathcal{C}$ is either the class of compact convex supports or the class of compact $\lambda$-convex supports. The notion of $\lambda$-convexity arises as a natural generalization of convexity, so every convex set is also $\lambda$-convex for all $\lambda>0$. We use the letter $\lambda$ here for convenience; other usual equivalent notations are $r$-convex or $\alpha$-convex. In short a set is $\lambda$-convex if it can be expressed as the intersection of the complements of a family of open balls with radii $\lambda$; see Perkal (1956), Walther $(1997,1999)$ and references therein.

\section{Some related literature}

In the recent paper by Berrendero, Cuevas, \& Pateiro-López (2011) a further uniformity test is proposed for the problem (1), when $\mathcal{C}$ is also the class of compact supports which are either convex or $\lambda$-convex. However the idea behind this test is completely different from that developed here as it is based on the size of the estimated maximal bivariate spacing (so we call it EMS test), as defined in Janson (1987). As we will see in the simulations below, the EMS procedure is, 
in some sense, complementary of the DBU test. While the former is particularly suitable for alternative hypothesis of Neyman-Scott type, e.g., for departures from uniformity which lead to "clustered observations", the DBU test turns out to be more powerful for "contamination models", prone to give more observations close to (or far away from) $\partial S$ than expected under uniformity.

In the work by Jain et al. (2002) it is analyzed (especially from the practical and computational point of view) another method for the uniformity testing problem with unknown support. It is based on ideas of graph theory.

Let us finally mention the interesting proposal by Liang et al. (2001). These authors propose a uniformity test, easy to implement even for very large dimensional data. However their method is designed for the specific case that $S=[0,1]^{d}$.

This paper is organized as follows. In Section 2, the basic ideas of the DB test proposed in Berrendero, Cuevas, \& Vázquez-Grande (2006) are summarized. Then the corresponding DBU test (for the case of unknown support) is defined. Also, some notions on $\lambda$-convex sets and their estimation are recalled. In Section 3 we show that the test statistic $D_{n}$ of the DB method and its counterpart $D_{n}^{*}$ in the new DBU procedure satisfy $\left|D_{n}-D_{n}^{*}\right| \rightarrow 0$, in probability, so that both tests are asymptotically equivalent regarding their properties of consistency and asymptotic preservation of the significance level. Section 4 is devoted to empirical results. Some geometric and computational issues are discussed in Section 5. The proofs are given in the Appendix.

\section{THE DBU TEST}

Let $S \subset \mathbb{R}^{2}$ be a compact set with non-empty interior. Let us also consider a two-dimensional random variable $X$ with support $S$ and denote by $\mathcal{X}_{n}=\left\{X_{1}, \ldots, X_{n}\right\}$ a sample drawn from $X$. As a first step in the development of our DBU test we briefly describe below the implementation of the original distance-to-boundary test with known support (DB test) proposed by Berrendero, Cuevas, \& Vázquez-Grande (2006).

The DB test: The support $S$ is known

The target is to test the null hypothesis

$H_{0}$ : the distribution of $X$ is uniform with support $S$.

Some notation: $D(x, y)$ denotes the Euclidean distance between points $x$ and $y$; for $A \subset \mathbb{R}^{2}$, $D(x, A)=\inf _{y \in A} D(x, y)$. The distribution function of the random variable $Y=D(X, \partial S)$ under $H_{0}$ will be denoted by $F$ and $\mathbb{F}_{n}$ is the empirical distribution function corresponding to $Y_{1}, \ldots, Y_{n}$, where $Y_{i}=D\left(X_{i}, \partial S\right)$ The usual Kolmogorov-Smirnov statistic is denoted by $D_{n}$, so that $D_{n}=\sqrt{n}\left\|F-\mathbb{F}_{n}\right\|$, where $\|\cdot\|$ stands for the sup-norm. The closed and open balls with centre $y$ and radius $r$ will be denoted respectively by $B(y, r)$ and $\stackrel{\circ}{B}(y, r)$.

Now, we are ready to recall the main ideas behind the DB test. In the study of this method it arises in a natural way a geometric condition on the support $S$ which is called "invariance by erosion upon translation" in Berrendero, Cuevas, \& Vázquez-Grande (2006). Roughly speaking, this condition, imposed on the set $S$, entails that the " $\epsilon$-eroded" versions of $S$, that is, the sets of type $\{x \in S: B(x, \epsilon) \subset S\}$ preserve the shape of $S$, in the sense that it coincides with $S$ except for an homothecy. Berrendero, Cuevas, \& Vázquez-Grande (2006) prove that any convex polygon circumscribed to a ball fulfills this condition. Essentially the same property is considered by Pegden (2011) which calls it "resiliency". This author proves a more general general result establishing that the sets resilient to erosion coincide with the convex bodies with an inscribed 
ball. We can summarize the implementation of the DB test as follows:

1. Given the original sample $X_{1}, \ldots, X_{n}$, compute the distances to the boundary $Y_{i}=$ $D\left(X_{i}, \partial S\right), i=1, \ldots, n$.

2. Compute the "maximum depth" $R=\max \{D(x, \partial S), x \in S\}$ and define the "normalized distances" $Y_{i}^{R}=Y_{i} / R$, for $i=1, \ldots, n$.

3. If the set $S$ is "invariant by erosion upon an homothecy" (see Berrendero, Cuevas, \& VázquezGrande (2006) for details and Pegden (2011) for closely related ideas) it can be proved that the distribution function $F^{R}$ of the $Y_{i}^{R}$, under $H_{0}$, is beta with parameters $a=1$ and $b=2$ (regardless of the support $S$ ). Then the DB test would reject $H_{0}$, at a level $\alpha$, if the KolmogorovSmirnov statistic based on the normalized distances $D_{n}^{R}=\sqrt{n}\left\|\mathbb{F}_{n}^{R}-F^{R}\right\|$ is greater that the corresponding critical value $D_{n, \alpha}$.

4. Otherwise (i.e., when $S$ does not fulfill the mentioned shape assumption), the distribution of the $Y_{i}^{R}$ will depend, in general, on $S$. So the normalization by $R$ indicated in the second step above is not particularly useful. In this case the test is performed, as indicated in the previous step, using the Kolmogorov-Smirnov statistic $D_{n}$ calculated from the (non-normalized) distances $Y_{i}$. If the distribution under $H_{0}$ of the $Y_{i}$ is difficult to calculate in a closed form it can be approximated by a Monte Carlo procedure by just drawing a large number of artificial iid observations $\hat{X}_{i}, i=1, \ldots, m$ from the uniform distribution on $S$ and taking the corresponding empirical distribution associated with $\hat{Y}_{i}=D\left(\hat{X}_{i}, \partial S\right)$ as an approximation to the distribution $F$.

\section{The DBU test: The support $S$ is unknown}

We next present the adaptation of the DB method for the case that the support $S$ is not specified in the null hypothesis. So, we will deal with the general problem (1) stated in the introduction. As commented above, the crucial idea is to replace the support $S$ with an appropriate support estimator $S_{n}=S_{n}\left(X_{1}, \ldots, X_{n}\right)$. There are all purpose set estimators which provide consistency properties (and even known convergence rates) under very general conditions on $S$; see Cuevas \& Fraiman (2009) for details. However, given the special role of $\partial S$ in the DB test, it is important for the plug-in estimator $\partial S_{n}$ to approximate the population counterpart at a fast enough rate. This will lead us to impose some restriction on the class $\mathcal{C}$ of possible supports. We will further comment on this below. Now, let us formally state the implementation of the DBU test:

1. Construct $S_{n}$, an estimator of $S$ based on the sample $\mathcal{X}_{n}$.

2. Define $\mathcal{X}_{n}^{*}=\left\{X_{1}^{*}, \ldots, X_{n^{*}}^{*}\right\}=\left\{X_{i} \in \mathcal{X}_{n}, X_{i} \notin \partial S_{n}, i=1, \ldots, n\right\}$.

3. Compute $Y_{i}^{*}=D\left(X_{i}^{*}, \partial S_{n}\right), i=1, \ldots, n^{*}$.

4. Let us consider a two-dimensional variable $\hat{X}$, uniform on $S_{n}$. The DBU test is based on the statistic $D_{n}^{*}=\sqrt{n}\left\|\mathbb{F}_{n}^{*}-\hat{F}\right\|$, where $\mathbb{F}_{n}^{*}$ is the empirical distribution of $Y_{i}^{*}=D\left(X_{i}^{*}, \partial S_{n}\right)$ and $\hat{F}$ is the distribution function of $\hat{Y}=D\left(\hat{X}, \partial S_{n}\right)$. Since this distribution under $H_{0}$ is difficult to calculate it is approximated by the empirical distribution of an artificial sample as described in the next step.

5. Generate an artificial sample $\hat{\mathcal{X}}_{m}=\left\{\hat{X}_{1}, \ldots, \hat{X}_{m}\right\}$, from a uniform distribution on the estimator $S_{n}$. Compute $\hat{Y}_{i}=D\left(\hat{X}_{i}, \partial S_{n}\right), i=1, \ldots, m$. Perform a two-sample KolmogorovSmirnov test of the null hypothesis that $Y_{i}^{*}$ and $\hat{Y}_{i}$ were drawn from the same continuous distribution.

The choice of the support estimator $S_{n}$ 
Keeping in mind that $S_{n}$ must provide and efficient, easy-to-compute estimator for both $S$ and (via $\partial S_{n}$ ) for $\partial S$, a natural choice for the class $\mathcal{C}$ in (1) would be

$$
\mathcal{C}=\left\{\text { class of compact convex supports with non-empty interior in } \mathbb{R}^{2}\right\} .
$$

In this case the natural estimator of $S$ is the convex hull of $\mathcal{X}_{n}$,

$$
S_{n}=\operatorname{conv}\left(\mathcal{X}_{n}\right)
$$

The properties of this estimator have been extensively analyzed since the early sixties; see Reitzner (2009). We will need here the consistency properties established by Dümbgen \& Walther (1996). In particular, these authors show that (for the two-dimensional case $d=2$ ), with probability 1 (a.s.),

$$
d_{H}\left(S_{n}, S\right)=\mathrm{O}\left(\left(\frac{\log n}{n}\right)^{1 / 2}\right)
$$

where $d_{H}(A, B)$ stands for the Hausdorff distance between two compact non-empty sets $A$ and $B$. As we will see, this convergence rate is not fast enough for our purposes. Under additional smoothness assumptions on $S$ (see also Walther (1997, 1999), Rodríguez-Casal (2007)) we have

$$
d_{H}\left(S_{n}, S\right)=\mathrm{O}\left(\left(\frac{\log n}{n}\right)^{2 / 3}\right), \text { a.s. }
$$

and, more importantly,

$$
d_{H}\left(\partial S_{n}, \partial S\right)=\mathrm{O}\left(\left(\frac{\log n}{n}\right)^{2 / 3}\right) \text {, a.s. }
$$

Whereas convexity is a simple, natural and well-studied assumption to be imposed on $S$, it is quite restrictive for many practical purposes. For example, when analyzing spatial patterns in ecological data, it is not always reasonable to assume that the habitat of a certain plant species is a convex domain. Hence we will also consider a second (much less popular) condition called $\lambda$-convexity with allows for a much more flexible class of possible supports. For another recent application of this condition to the problem of testing uniformity see Berrendero, Cuevas, \& Pateiro-López (2011).

A closed set $S \subset \mathbb{R}^{2}$ is said to be $\lambda$-convex for some $\lambda>0$ if $S$ coincides with its $\lambda$-convex hull, that is $S=C_{\lambda}(S)$, where

$$
C_{\lambda}(S)=\bigcap_{\dot{B}(y, \lambda) \cap S=\emptyset} \stackrel{\circ}{B}(y, \lambda)^{c} .
$$

In other words, $S$ can be expressed as the intersection of the complements of a family of open balls with radii $\lambda$. The origin of this notion goes back to Perkal (1956). See Walther (1997), Rodríguez-Casal (2007), Berrendero, Cuevas, \& Pateiro-López (2011), and references therein, for additional insights as well as statistical applications.

The condition of $\lambda$-convexity is clearly reminiscent of the plain notion of convexity, as it can be seen by replacing the balls in (4) by half-spaces. In fact, every closed convex set is also $\lambda$-convex for all $\lambda>0$. It is also apparent that $\lambda$-convexity is a much more flexible condition 
which allows the set to have inlands (as long as they are not too sharp) or holes and even to be disconnected.

From a statistical point of view, the most important feature of definition (4) is the fact that a $\lambda$-convex support $S$ has a natural estimator from a random sample $\mathcal{X}_{n}$ which is the $\lambda$-convex hull of the sample points,

$$
S_{n}=C_{\lambda}\left(\mathcal{X}_{n}\right)
$$

This estimator turns out to be computationally feasible; the R-package alphahull developed by Pateiro-López \& Rodríguez-Casal (2010) provides an efficient calculation of (5) in the twodimensional case. Moreover, under appropriate smoothness conditions, this estimator exhibits also the fast convergence rates (2) and (3). This will be important in the theoretical developments of the following section.

\section{ASYMPTOTIC PROPERTIES}

The aim of this section is to show that, under suitable shape restrictions on the class $\mathcal{C}$ in (1), the DBU test is asymptotically equivalent to the DB test proposed in Berrendero, Cuevas, \& Vázquez-Grande (2006) for the case of a known support. According to the notation introduced in Section 2, this amounts to show that $\left|D_{n}-D_{n}^{*}\right| \rightarrow 0$, in probability as $n \rightarrow \infty$. We will establish this in two results (Theorems 1 and 2 below), obtained under two different assumptions for $\mathcal{C}$.

We introduce some notation for the results and their proofs. In what follows, $\mathcal{X}_{n}=$ $\left\{X_{1}, \ldots, X_{n}\right\}$ will denote a sample drawn on a compact support $S$, with non-empty interior. The Lebesgue measure of a set $A$ will be denoted by $\mu(A)$ and the cardinal of the set $\left\{i: X_{i} \in \partial S_{n}\right\}$ will be denoted by $N_{n}$ (that is, $N_{n}=n-n^{*}$ ). All the convergence results below correspond to limits as $n \rightarrow \infty$.

The proofs are organized in three lemmas and two theorems, see the Appendix. The general structure is as follows. Lemma 1 establishes the asymptotic proximity (with a $\sqrt{n}$ rate) of $\hat{F}$ to $F$. Lemma 2 proves an analogous result for $\mathbb{F}_{n}$ and $\mathbb{F}_{n}^{*}$. Lemma 3 establishes (as a direct consequence of the two previous lemmas) the asymptotic equivalence of test statistics $D_{n}$ and $D_{n}^{*}$. The practical conclusion is the asymptotic equivalence of the DB and the DBU test. Finally, Theorems 1 and 2 show that the conclusion of Lemma 3 can be applied to the case of convex support (Theorem 1) and to the more general assumption of $\lambda$-convex support (Theorem 2).

Lemma 1. Assume that the support $S$ and the estimator $S_{n}$ are such that $F$ is Lipschitz continuous, $S_{n} \subset S$ with probability one, $\sqrt{n} \mu\left(S \backslash S_{n}\right) \stackrel{P}{\rightarrow} 0$ and $\sqrt{n} d_{H}\left(\partial S, \partial S_{n}\right) \stackrel{P}{\rightarrow} 0$. Then, $\sqrt{n}\|\hat{F}-F\| \stackrel{P}{\rightarrow} 0$.

Lemma 2. Assume that the support $S$ and the estimator $S_{n}$ are such that $F$ is Lipschitz continuous, $\mathcal{X}_{n} \subset S_{n} \subset S$ with probability one, $N_{n} / \sqrt{n} \stackrel{P}{\rightarrow} 0$ and $n^{1 / 2+\delta} d_{H}\left(\partial S, \partial S_{n}\right) \stackrel{\text { a.s. }}{\rightarrow} 0$, for some $\delta>0$. Then, $\sqrt{n}\left\|\mathbb{F}_{n}^{*}-\mathbb{F}_{n}\right\| \stackrel{P}{\rightarrow} 0$.

Lemma 3. Assume that the support $S$ and the estimator $S_{n}$ are such that $F$ is Lipschitz continuous, $\mathcal{X}_{n} \subset S_{n} \subset S$ with probability one, $\sqrt{n} \mu\left(S \backslash S_{n}\right) \stackrel{P}{\rightarrow} 0, N_{n} / \sqrt{n} \stackrel{P}{\rightarrow} 0$, and $n^{1 / 2+\delta} d_{H}\left(\partial S, \partial S_{n}\right) \stackrel{a . s .}{\rightarrow} 0$, for some $\delta>0$. Then, $\left|D_{n}-D_{n}^{*}\right| \stackrel{P}{\rightarrow} 0$.

Now we apply Lemma 3 to the cases when we can assume that $S$ is convex and $\lambda$-convex respectively. We will also need the following smoothness condition: A ball of radius $r$ is said to roll freely inside a closed set $A \subset \mathbb{R}^{d}$ if for each point $a \in \partial A$ there exists $x \in \mathbb{R}^{d}$ such that $a \in B(x, r) \subset A$. 
Theorem 1. Let $S \subset \mathbb{R}^{2}$ be a compact convex set with nonempty interior such that $F$ is Lipschitz continuous and such that a ball of radius $r>0$ rolls freely inside $S$ for some $r>0$. Let $S_{n}$ be the convex hull of $\mathcal{X}_{n}$. Assume further that the $X_{i}$ have a common Lebesque density bounded away from zero on $S$. Then $\left|D_{n}-D_{n}^{*}\right| \stackrel{P}{\rightarrow} 0$.

Theorem 2. Let $S \subset \mathbb{R}^{2}$ be a compact $\lambda$-convex set with nonempty interior such that $\overline{S^{c}}$ is also $\lambda$-convex and $\operatorname{int}\left(S_{i}\right) \neq \emptyset$ for each path-connected component $S_{i} \subset S$. Assume that $F$ is Lipschitz continuous. Let $S_{n}$ be the $\lambda$-convex hull of $\mathcal{X}_{n}$. Assume further that the $X_{i}$ have a common Lebesque density bounded away from zero on $S$. Then $\left|D_{n}-D_{n}^{*}\right| \stackrel{P}{\rightarrow} 0$.

As a consequence of these results the DBU test inherits the properties of the DB test studied in Berrendero, Cuevas, \& Vázquez-Grande (2006), in particular, (under the conditions of Theorems 1 or 2) it asymptotically preserves the prescribed significance level and both tests are consistent to detect the same non-uniform alternatives.

\section{EMPIRICAL RESULTS}

\subsection{Empirical significance level}

We have checked the performance of the DBU test in terms of preservation of the nominal confidence level. The numerical results given below have been obtained using the $\mathrm{R}$ software, see $\mathrm{R}$ Development Core Team (2011).

A simulation example: the "unknown" support $S$ is a set limited by a Lamé curve. The possible supports in the null hypothesis are either convex or $\lambda$-convex

Table 1 gives the outputs corresponding to the empirical significance level obtained (as an average over 5000 independent runs) with the DBU test and the DB test intended for nominal significance levels $\alpha=0.05,0.1$. Sample sizes are $n=50,100,200$. The considered supports are sets limited by different Lamé curves (also called superellipses), that is, sets of the form $S=\left\{(x, y) \in \mathbb{R}^{2}:|x|^{r}+|y|^{r} \leq 1\right\}$ for different values of $r$, see Figure 1. Note that for $r=1$ and $r=2$ the equation of the Lamé curve describes a square and a circle, respectively. We refer to Jaklič, Leonardis, \& Solina (2000) for further discussion of superellipses and their properties.

The supports limited by these curves for $r=1$ and $r=2$ are invariant by erosion upon an homothecy (see Section 2 above for more details on this). Thus we are under the assumptions of Theorem 1 in Berrendero, Cuevas, \& Vázquez-Grande (2006) for the DB test, so that the distribution of $Y^{R}=D(X, \partial S) / R$ under the null hypothesis is totally known (it is a beta distribution $\beta(1,2)$ ) and we may perform a classical one-sample Kolmogorov-Smirnov test of goodness of fit to that distribution.

For other values of $r$, the set $S$ does not fulfill the mentioned shape restriction and the distribution of $Y$ is derived in practice by a Monte Carlo mechanism; see the description of the implementation of the test in Section 2. Moreover, the non-normalized distances $Y_{i}$ in the DB test are approximated numerically, since there is no solution in closed form for the distance to the Lamé curve when $r=3$ or $r=4$, see Rosin \& West (1995). For the DBU test we use as estimator $S_{n}$ both the convex hull of the sample $\mathcal{H}\left(\mathcal{X}_{n}\right)$ and the $\lambda$-convex hull of the sample $C_{\lambda}\left(\mathcal{X}_{n}\right)$ (with $\lambda=1$ ). This corresponds to take $\mathcal{C}$ in the null hypothesis (1) to be the class of compact convex sets or the class of compact $\lambda$-convex sets, respectively. 

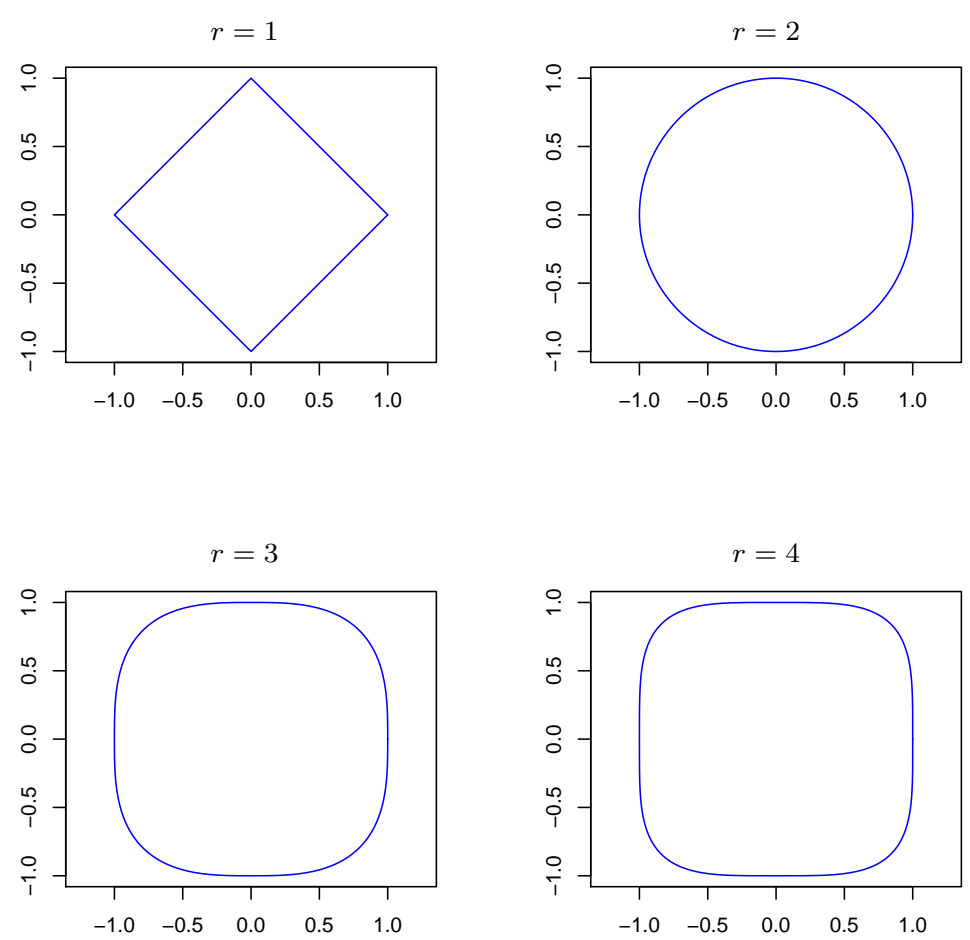

FiguRE 1: Lamé curves $|x|^{r}+|y|^{r}=1$ for different values of $r$.

The slight (non-systematic) improvements observed in some cases in the DBU test (with respect to the DB test) can be explained by the fact that, on average, the DBU procedure underestimates the proportion of observations near the boundary, since the points in the boundary of the convex hull $\mathcal{H}\left(\mathcal{X}_{n}\right)$ and those in the boundary of the $\lambda$-convex hull, $C_{\lambda}\left(\mathcal{X}_{n}\right)$, are excluded from consideration. So, in the DBU test those uniform samples that, by chance, turn out to be unusually close to the boundary (which therefore would tend to increase the type I error) are less likely to appear in the DBU procedure. Of course, as a counterpart, there is an obvious effect against the DBU procedure since the samples under the null hypothesis are drawn from $S$ and DBU tests in fact the uniformity on $S_{n}$. The oscillations in the performance of DBU and DB represent the balance between both opposite effects.

A case with non-connected support

Let $S$ be the set in Figure 2, which is not convex but $\lambda$-convex for $\lambda=2$. Table 2 gives the outputs corresponding to the empirical significance level obtained (as an average over 10000 independent runs) with the DBU test and the DB test intended for nominal significance levels $\alpha=0.05,0.1$. 
TABLE 1: Empirical significance level of the DBU test and DB test over 5000 uniform samples of size $n=50,100,200$ on the supports $S=\left\{(x, y) \in \mathbb{R}^{2}:|x|^{r}+|y|^{r} \leq 1\right\}$ for different values of $r$. The nominal values are $0.05,0.1$. For the DBU test, we consider $S_{n}=\mathcal{H}\left(\mathcal{X}_{n}\right)$ and $S_{n}=C_{\lambda}\left(\mathcal{X}_{n}\right)$ with $\lambda=1$.

\begin{tabular}{|c|c|c|c|c|c|c|c|c|}
\hline & & \multicolumn{3}{|c|}{$\begin{array}{c}\text { DBU test } \\
S_{n}=\mathcal{H}\left(\mathcal{X}_{n}\right)\end{array}$} & \multicolumn{2}{|c|}{$\begin{array}{c}\text { DBU test } \\
S_{n}=C_{\lambda}\left(\mathcal{X}_{n}\right)\end{array}$} & \multicolumn{2}{|c|}{ DB test } \\
\hline & & $\alpha$ & 0.05 & 0.1 & 0.05 & 0.1 & 0.05 & 0.1 \\
\hline \multirow[t]{3}{*}{$r=1$} & $n=50$ & & 0.0436 & 0.0868 & 0.0422 & 0.0860 & 0.0460 & 0.0896 \\
\hline & $n=100$ & & 0.0414 & 0.0888 & 0.0406 & 0.0858 & 0.0408 & 0.0834 \\
\hline & $n=200$ & & 0.0492 & 0.0962 & 0.0476 & 0.0960 & 0.0418 & 0.0864 \\
\hline \multirow[t]{3}{*}{$r=2$} & $n=50$ & & 0.0474 & 0.0906 & 0.0400 & 0.0866 & 0.0472 & 0.0940 \\
\hline & $n=100$ & & 0.0416 & 0.0828 & 0.0452 & 0.0920 & 0.0450 & 0.0934 \\
\hline & $n=200$ & & 0.0510 & 0.0934 & 0.0490 & 0.0966 & 0.0522 & 0.1018 \\
\hline \multirow[t]{3}{*}{$r=3$} & $n=50$ & & 0.0502 & 0.0974 & 0.0482 & 0.0932 & 0.0500 & 0.0954 \\
\hline & $n=100$ & & 0.0468 & 0.0902 & 0.0442 & 0.0902 & 0.0472 & 0.0938 \\
\hline & $n=200$ & & 0.0480 & 0.0998 & 0.0510 & 0.1016 & 0.0444 & 0.0890 \\
\hline \multirow[t]{3}{*}{$r=4$} & $n=50$ & & 0.0416 & 0.0820 & 0.0376 & 0.0790 & 0.0414 & 0.0834 \\
\hline & $n=100$ & & 0.0448 & 0.0920 & 0.0432 & 0.0880 & 0.0428 & 0.0846 \\
\hline & $n=200$ & & 0.0470 & 0.0946 & 0.0468 & 0.0908 & 0.0344 & 0.0718 \\
\hline
\end{tabular}

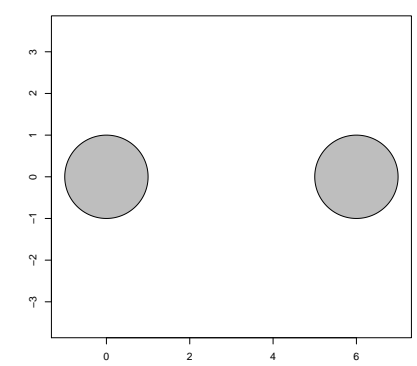

FIGURE 2: Non-convex support $S=B(x, 1) \cup B(y, 1)$, with $x=(0,0)$ and $y=(6,0)$. The set $S$ is not convex but $\lambda$-convex for $\lambda=2$.

Some results in $\mathbb{R}^{3}$

We have also studied the behavior in terms of significance level of the DB test and DBU test in $\mathbb{R}^{3}$. The algorithms are essentially the same as those described in Section 2. Table 3 gives the outputs corresponding to the empirical significance level obtained (as an average over 10000 independent runs) with the DBU test and the DB test intended for nominal significance levels $\alpha=0.05,0.1$. Sample sizes are $n=50,100,200,500$. The considered supports are the unit cube $S=[0,1]^{3}$ and unit ball $S=B(0,1)$ in $\mathbb{R}^{3}$. Since both supports are invariant by erosion 
TABLE 2: Empirical significance level of the DBU test and DB test over 10000 uniform samples of size $n=50,100,200,500$ on $S$ in Figure 2. The nominal values are 0.05, 0.1. For the DBU test, we consider $S_{n}=C_{\lambda}\left(\mathcal{X}_{n}\right)$ with $\lambda=2$.

\begin{tabular}{|c|c|c|c|c|c|}
\hline & \multicolumn{3}{|c|}{$\begin{array}{c}\text { DBU test } \\
S_{n}=C_{\lambda}\left(\mathcal{X}_{n}\right)\end{array}$} & \multicolumn{2}{|c|}{ DB test } \\
\hline & $\alpha$ & 0.05 & 0.1 & 0.05 & 0.1 \\
\hline$n=50$ & & 0.0433 & 0.0860 & 0.0480 & 0.0981 \\
\hline$n=100$ & & 0.0472 & 0.0933 & 0.0437 & 0.0922 \\
\hline$n=200$ & & 0.0439 & 0.0934 & 0.0417 & 0.0889 \\
\hline$n=500$ & & 0.0466 & 0.0967 & 0.0495 & 0.0948 \\
\hline
\end{tabular}

upon an homothecy we perform for the DB test a classical one-sample Kolmogorov-Smirnov test of the null that the distribution function of the random variable $Y^{R}=D(X, \partial S) / R$ is a beta distribution with parameters $a=1$ and $b=3$. For the DBU test, we restrict ourselves to the case where the support $S$ is assumed to be convex and is estimated through the convex hull of the sample $\mathcal{H}\left(\mathcal{X}_{n}\right)$.

TABLE 3: Empirical significance level of the DBU test and DB test over 10000 uniform samples of size $n=50,100,200,500$ on $S=[0,1]^{3}$ and $S=B(0,1)$ in $\mathbb{R}^{3}$. The nominal values are $0.05,0.1$. For the DBU test, we consider $S_{n}=\mathcal{H}\left(\mathcal{X}_{n}\right)$.

\begin{tabular}{|c|c|c|c|c|c|c|}
\hline & & \multicolumn{3}{|c|}{$\begin{array}{c}\text { DBU test } \\
S_{n}=\mathcal{H}\left(\mathcal{X}_{n}\right)\end{array}$} & \multicolumn{2}{|c|}{ DB test } \\
\hline & & $\alpha$ & 0.05 & 0.1 & 0.05 & 0.1 \\
\hline \multirow[t]{4}{*}{$S=[0,1]^{3}$} & $n=50$ & & 0.0399 & 0.0831 & 0.0483 & 0.0969 \\
\hline & $n=100$ & & 0.0434 & 0.0886 & 0.0423 & 0.0872 \\
\hline & $n=200$ & & 0.0468 & 0.0916 & 0.0487 & 0.0950 \\
\hline & $n=500$ & & 0.0470 & 0.0938 & 0.0483 & 0.0956 \\
\hline \multirow[t]{4}{*}{$S=B(0,1)$} & $n=50$ & & 0.0378 & 0.0798 & 0.0480 & 0.0972 \\
\hline & $n=100$ & & 0.0453 & 0.0886 & 0.0488 & 0.0968 \\
\hline & $n=200$ & & 0.0449 & 0.0909 & 0.0433 & 0.0872 \\
\hline & $n=500$ & & 0.0510 & 0.0970 & 0.0507 & 0.0948 \\
\hline
\end{tabular}

\subsection{Power study}

As for the power study, we have considered two different models in the choice of the alternative distribution.

\section{Contamination model}



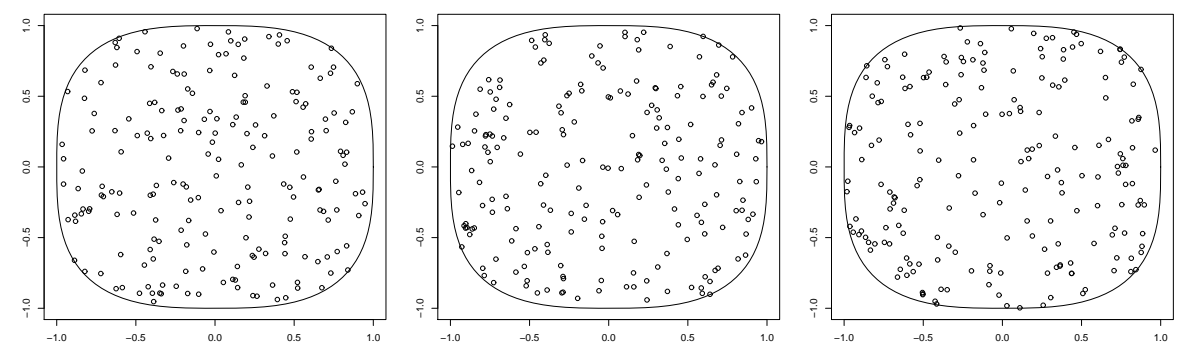

FIGURE 3: Random samples of size $n=200$ from mixtures of type $(1-\epsilon) U(S)+\epsilon U\left(S \backslash S_{0}\right)$, where $S=\left\{(x, y) \in \mathbb{R}^{2}:|x|^{r}+|y|^{r} \leq 1\right\}$ for $r=3$ and $S_{0}$ denotes a set like $S$ with the same centre and area $\mu(S) / 2$. Left, $\epsilon=0.1$. Middle, $\epsilon=0.2$. Right, $\epsilon=0.3$.

The sample points are drawn from a random variable whose distribution is given by a mixture of type $(1-\epsilon) U(S)+\epsilon U\left(S \backslash S_{0}\right)$, where $S=\left\{(x, y) \in \mathbb{R}^{2}:|x|^{r}+|y|^{r} \leq 1\right\}$ for $r=3$ and $S_{0}$ denotes a set like $S$ with the same centre and area $\mu(S) / 2$. We have taken $\epsilon=0.1,0.2,0.3$, see Figure 3. We have compared the performance of the DBU test with that of the EMS test (based on multivariate spacings) by Berrendero, Cuevas, \& Pateiro-López (2011). The corresponding outputs are summarized in Table 4.

TABLE 4: Empirical powers over 5000 runs of the DBU test, EMS test and DB test. The underlying distributions are contaminated uniforms $(1-\epsilon) U(S)+\epsilon U\left(S \backslash S_{0}\right)$, where $S=\left\{(x, y) \in \mathbb{R}^{2}:|x|^{r}+|y|^{r} \leq 1\right\}$ for $r=3$ and $S_{0}$ denotes a set like $S$ with the same centre and area $\mu(S) / 2$. The significance level is 0.05 .

\begin{tabular}{|c|c|c|c|c|c|}
\hline & & $\begin{array}{c}\text { DBU test } \\
S_{n}=\mathcal{H}\left(\mathcal{X}_{n}\right)\end{array}$ & $\begin{array}{c}\text { DBU test } \\
S_{n}=C_{\lambda}\left(\mathcal{X}_{n}\right)\end{array}$ & DB test & EMS test \\
\hline \multirow[t]{3}{*}{$\epsilon=0.1$} & $n=50$ & 0.0646 & 0.0598 & 0.1078 & 0.0130 \\
\hline & $n=100$ & 0.1042 & 0.0974 & 0.1716 & 0.0404 \\
\hline & $n=200$ & 0.2028 & 0.1934 & 0.3112 & 0.0566 \\
\hline \multirow[t]{3}{*}{$\epsilon=0.2$} & $n=50$ & 0.1438 & 0.1168 & 0.2584 & 0.0212 \\
\hline & $n=100$ & 0.3346 & 0.2990 & 0.4786 & 0.0638 \\
\hline & $n=200$ & 0.6110 & 0.5844 & 0.7778 & 0.0992 \\
\hline \multirow[t]{3}{*}{$\epsilon=0.3$} & $n=50$ & 0.3218 & 0.2570 & 0.5118 & 0.0374 \\
\hline & $n=100$ & 0.6598 & 0.6178 & 0.8176 & 0.1126 \\
\hline & $n=200$ & 0.9456 & 0.9338 & 0.9852 & 0.1910 \\
\hline
\end{tabular}

Neyman-Scott clustering alternatives 
This is a typical deviation from the uniformity assumption, often considered in the theory of point processes. Under this model the sample tends to provide "clustered" observations. For the simulated samples each cluster consist of $m$ points, generated from the uniform distribution on a disc of radius $r$, which entails a departure from the iid assumption for the data. The corresponding outputs are summarized in Table 5.

The support estimator used in the second column of Table 4 and in Table 5 is $S_{n}=C_{\lambda}\left(\mathcal{X}_{n}\right)$ with $\lambda=1$.

TABLE 5: Empirical powers of the uniformity tests under study over 5000 runs of sample size $n=100$ and $n=200$ from Neyman-Scott clustering alternatives. Each cluster consist of $m$ points, generated from the uniform distribution on a disc of radius $r$.

\begin{tabular}{lllccc}
\hline & & & DBU test & DB test & EMS test \\
\hline$r=0.05$ & $m=5$ & $n=100$ & 0.6174 & 0.4946 & 0.9790 \\
& & $n=200$ & 0.5608 & 0.4976 & 0.9976 \\
$r=0.05$ & $m=10$ & $n=100$ & 0.9030 & 0.7556 & 0.9994 \\
& & $n=200$ & 0.8504 & 0.7560 & 1.0000 \\
$r=0.1$ & $m=5$ & $n=100$ & 0.3458 & 0.3668 & 0.7952 \\
& & $n=200$ & 0.3338 & 0.3670 & 0.8442 \\
$r=0.1$ & $m=10$ & $n=100$ & 0.5784 & 0.5822 & 0.9828 \\
& & $n=200$ & 0.5404 & 0.5746 & 0.9970 \\
\hline
\end{tabular}

In order to properly interpret these results one should keep in mind that the Neyman-Scott model does not correspond to the case of independent identically distributed observations. Thus, depending on the number of clusters $m$ and the radius $r$ we could find that the lack of uniformity in this model is harder to detect with larger samples. The reason is that for large samples one would have a larger number of clusters whose centres are uniformly distributed so giving a false appearance of uniformity.

Also, it can be observed that the DBU test outperforms DB when the cluster radius is small. This can be explained by the "boundary effect" present in the DBU method. Recall again that the points in the boundary of the support estimator are taken out but, under the Neyman-Scott model, all these excluded points have a cluster of close (when $r$ is small) non-excluded points near the boundary. These points help us to detect the lack of uniformity.

\subsection{Conclusions}

1. The results in Tables 1 and 2 show that the DBU test succeeds in preserving the significance level (though it tends to be slightly conservative). The cost of estimating the support (pointed out by the difference observed with the DB test, where the support is known) turns out to be moderate and quite affordable in statistical terms.

2. Note that the asymptotic validity of the DBU test in the tri-dimensional case is not covered by our theoretical results in Section 3 (which apply only for $d=2$ ). However, the outputs in Table 3 suggest that the method could work even in this case. A new, quite different, theoretical approach would be needed in this case, as the arguments in Section 3 rely essentially on the assumption $d=2$. From the computational point of view, the implementation of the 
DBU test presents some technical difficulties for $d=3$. The convex hull estimator can be computed in general dimension, see for example the R-package geometry by Grasman \& Gramacy (2010). However, the $\lambda$-convex hull is only implemented in the bi-dimensional case. This practical restriction forces us to consider convex supports and the convex hull estimator in Table 3. A possible solution for non-convex supports in $\mathbb{R}^{3}$ would be to compute the $\lambda$ shape, see Edelsbrunner \& Mücke (1994). The $\lambda$-shape is computationally practicable, and it is closely related to the $\lambda$-convex hull estimator (it approximates the boundary of the $\lambda$-convex hull by a piecewise linear surface). The implementation in $\mathrm{R}$ of this structure is currently under development.

3. The power results in Tables 4 and 5 show also a foreseeable behavior: the procedure works efficiently for detecting "contaminated" distributions but it is much less powerful for NeymanScott alternatives. Again, the loss of efficiency associated with the estimation of the support is surprisingly low. As mentioned in the Introduction, the "spacing-based" EMS procedure (see Berrendero, Cuevas, \& Pateiro-López (2011) for details) can be thought as complementary to the DBU test. The EMS test is suitable for alternative hypothesis that provide "clustered" observations but it is less powerful for "contamination models", where the DBU test shows a clear superiority. The slight loss of power observed when increasing the sample size in some cases in Table 5 may be explained by the dependence of the observations generated from the Neyman-Scott model.

\section{GENERATION OF UNIFORM SAMPLES ON $S_{N}$}

The uniformity test for the case of an unknown support $S$ is based on the statistic

$$
D_{n}^{*}=\sqrt{n}\left\|\mathbb{F}_{n}^{*}-\hat{F}\right\|,
$$

being $\hat{F}$ the distribution of the random variable $\hat{Y}=D\left(\hat{X}, \partial S_{n}\right)$, where $\hat{X}$ is uniform on $S_{n}$. Since $\hat{F}$ is unknown, this distribution is derived in practice by a Monte Carlo mechanism. A large number of iid uniform observations $\hat{X}_{i}, i=1, \ldots, m$ are drawn on $S_{n}$. The empirical distribution corresponding to the sample $\hat{Y}_{i}=D\left(\hat{X}_{i}, \partial S_{n}\right), i=1, \ldots, m$ is used as approximation for $\hat{F}$.

\section{Uniform samples on $\mathcal{H}\left(\mathcal{X}_{n}\right)$.}

Assume that we choose as estimator $S_{n}=\mathcal{H}\left(\mathcal{X}_{n}\right)$. The problem of how to generate uniform random vectors on the convex hull of a set of points in $\mathbb{R}^{2}$ is well-known. Note that this is a particular case of uniform random generation on a convex polygon in the plane, which is solved by means of triangulation. See Devroye (1986) for a description of the algorithm. The procedure in $\mathbb{R}^{3}$ is similar. In this case, we partition the convex hull of the sample into tetrahedra by means of the Delaunay triangulation of the sample, which can be computed in R by means of the library geometry, see Grasman \& Gramacy (2010). To generate a point uniformly in the triangulated polyhedron, we first sample one of the tetrahedra with probabilities proportional to their volumes and then we sample a point uniformly in the selected tetrahedron. The generation of uniform random vectors in a tetrahedron is a particular case of the generation of uniform random vectors in a simplex for dimension $d=3$. See Figure 4.

Uniform samples on $C_{\lambda}\left(\mathcal{X}_{n}\right)$. 

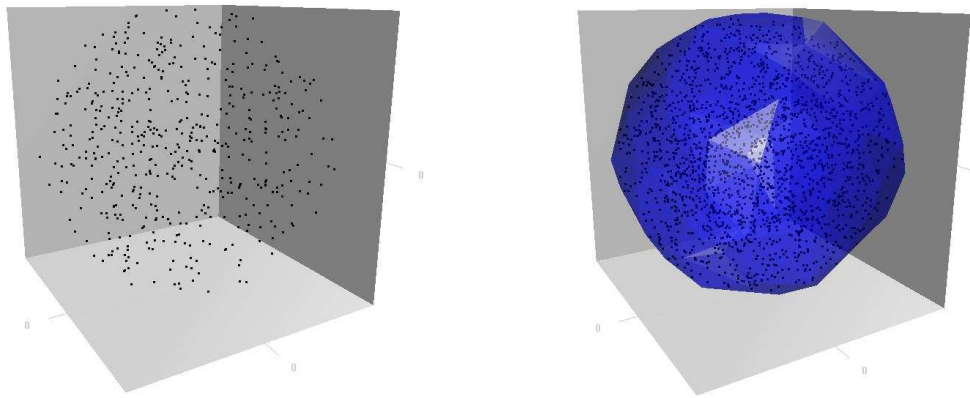

FIGURE 4: Uniform sample $\mathcal{X}_{n}$ in $B(0,1)$ in $\mathbb{R}^{3}$ of size $n=500$ (left). Convex hull $\mathcal{H}\left(\mathcal{X}_{n}\right)$ and uniform sample generated on $\mathcal{H}\left(\mathcal{X}_{n}\right)$ of size $m=2000$ (right).

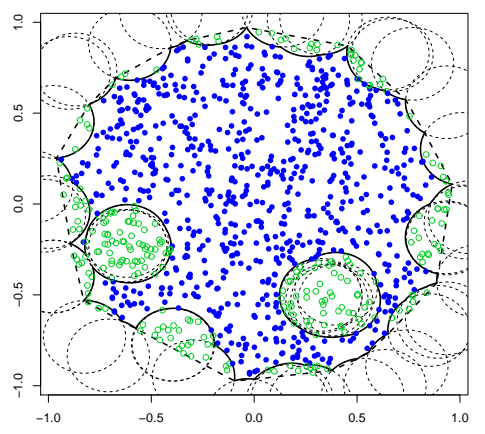

FIGURE 5: Uniform sample (solid points) in $C_{\lambda}\left(\mathcal{X}_{n}\right)$. The sample is obtained from uniform observations in the convex hull $\mathcal{H}\left(\mathcal{X}_{n}\right)$ after removing the sample points (non-solid points) that belong to any of the balls defining the complement of the $\lambda$-convex hull.

Assume now that $S_{n}=C_{\lambda}\left(\mathcal{X}_{n}\right)$. In order to generate uniform samples on $C_{\lambda}\left(\mathcal{X}_{n}\right)$ we proceed as follows: first, we generate a large sample of uniform observations in the convex hull $\mathcal{H}\left(\mathcal{X}_{n}\right)$. Note that the $\lambda$-convex hull is contained in the convex hull. Then, we remove the points that belong to any of the balls defining the complement of the $\lambda$-convex hull. The resulting sample is uniform in $C_{\lambda}\left(\mathcal{X}_{n}\right)$, see Figure 5.

\section{APPENDIX}

Proof of Lemma 1. Since both $F$ and $\hat{F}$ have compact support, there exists $K>0$ (not depending on $n$ ) such that $\|\hat{F}-F\|=\sup _{t \in[0, K]}|\hat{F}(t)-F(t)|$. Let $B$ be the closed unit ball in $\mathbb{R}^{2}$ and denote by $C \ominus D=\{x: x+D \subset C\}$ the Minkowski difference of two sets $C$ and $D$. Observe that $Y \geq t$ if and only if $X \in S \ominus t B$, and $\hat{Y} \geq t$ if and only if $\hat{X} \in S_{n} \ominus t B$. Then,

$$
|\hat{F}(t)-F(t)|=\left|\mathbb{P}\left(\hat{X} \in S_{n} \ominus t B\right)-\mathbb{P}(X \in S \ominus t B)\right|,
$$


and, using the triangle inequality,

$$
\begin{aligned}
|\hat{F}(t)-F(t)| & \leq\left|\mathbb{P}\left(\hat{X} \in S_{n} \ominus t B\right)-\mathbb{P}\left(X \in S_{n} \ominus t B\right)\right| \\
& +\left|\mathbb{P}\left(X \in S_{n} \ominus t B\right)-\mathbb{P}(X \in S \ominus t B)\right| .
\end{aligned}
$$

Regarding the first term in the right-hand side of inequality (1), observe that, for all $t \in[0, K]$,

$$
\begin{aligned}
& \left|\mathbb{P}\left(\hat{X} \in S_{n} \ominus t B\right)-\mathbb{P}\left(X \in S_{n} \ominus t B\right)\right|=\frac{\mu\left(S_{n} \ominus t B\right)}{\mu\left(S_{n}\right)}-\frac{\mu\left(S_{n} \ominus t B\right)}{\mu(S)} \\
& =\frac{\mu\left(S_{n} \ominus t B\right)}{\mu\left(S_{n}\right)}\left(1-\frac{\mu\left(S_{n}\right)}{\mu(S)}\right) \leq 1-\frac{\mu\left(S_{n}\right)}{\mu(S)}=\frac{\mu\left(S \backslash S_{n}\right)}{\mu(S)} .
\end{aligned}
$$

Since, by assumption, $\sqrt{n} \mu\left(S \backslash S_{n}\right) \stackrel{\mathrm{P}}{\rightarrow} 0$, we also have

$$
\sqrt{n} \sup _{t \in[0, K]}\left|\mathbb{P}\left(\hat{X} \in S_{n} \ominus t B\right)-\mathbb{P}\left(X \in S_{n} \ominus t B\right)\right| \stackrel{\mathrm{P}}{\rightarrow} 0 .
$$

For the second term in the right-hand side of inequality (1), observe that, for all $t \in[0, K]$,

$$
\mathbb{P}\left(X \in(S \ominus t B) \backslash\left(S_{n} \ominus t B\right)\right) \leq \mathbb{P}\left(Y \geq t, D\left(X, \partial S_{n}\right)<t\right)+\frac{\mu\left(S \backslash S_{n}\right)}{\mu(S)},
$$

since $X \in S \ominus t B$ amounts to $Y \geq t$, and $X \notin S_{n} \ominus t B$ implies that $D\left(X, \partial S_{n}\right)<t$ or $X \in$ $S \backslash S_{n}$.

Also, $D\left(X, \partial S_{n}\right)<t$ implies $Y<t+\epsilon_{n}$, where $\epsilon_{n}=d_{H}\left(\partial S, \partial S_{n}\right)$. Indeed, since $D\left(X, \partial S_{n}\right)<t$, there exists $z_{n} \in \partial S_{n}$ such that $D\left(X, z_{n}\right)<t$. By definition of Hausdorff distance, there exists $z \in \partial S$ with $D\left(z, z_{n}\right) \leq \epsilon_{n}$. Hence,

$$
Y=D(X, \partial S) \leq D(X, z) \leq D\left(X, z_{n}\right)+D\left(z_{n}, z\right)<t+\epsilon_{n} .
$$

As a consequence,

$$
\mathbb{P}\left(X \in(S \ominus t B) \backslash\left(S_{n} \ominus t B\right)\right) \leq \mathbb{P}\left(t \leq Y<t+\epsilon_{n}\right)+\frac{\mu\left(S \backslash S_{n}\right)}{\mu(S)} .
$$

Since $F$ is Lipschitz continuous, there exists $M>0$ such that $\mathbb{P}\left(t \leq Y<t+\epsilon_{n}\right)=F(t+$ $\left.\epsilon_{n}\right)-F(t) \leq M \epsilon_{n}$. From this bound, (2) and taking into account the assumptions we deduce

$$
\sqrt{n} \sup _{t \in[0, K]} \mathbb{P}\left(X \in(S \ominus t B) \backslash\left(S_{n} \ominus t B\right)\right) \leq M \sqrt{n} \epsilon_{n}+\frac{\sqrt{n} \mu\left(S \backslash S_{n}\right)}{\mu(S)} \stackrel{\mathrm{P}}{\rightarrow} 0 .
$$

Proof of Lemma 2. For $i=1, \ldots, n$ define $\tilde{Y}_{i}=D\left(X_{i}, \partial S_{n}\right)$ and let $\tilde{\mathbb{F}}_{n}$ be the empirical distribution function corresponding to $\tilde{Y}_{1}, \ldots, \tilde{Y}_{n}$. Since

$$
\sqrt{n}\left\|\mathbb{F}_{n}^{*}-\mathbb{F}_{n}\right\| \leq \sqrt{n}\left\|\mathbb{F}_{n}^{*}-\tilde{\mathbb{F}}_{n}\right\|+\sqrt{n}\left\|\tilde{\mathbb{F}}_{n}-\mathbb{F}_{n}\right\|
$$

it is enough to prove that both terms in the right-hand side of the last inequality go to zero in probability. Since there exists $K>0$ such that all the involved distributions have supports included in $[0, K]$, the sup-norms can always be computed on a compact interval $[0, K]$ instead of $\mathbb{R}$.

DOI: 
Observe that, for $t \geq 0, \tilde{\mathbb{F}}_{n}(t)=\left(1-N_{n} / n\right) \mathbb{F}_{n}^{*}(t)+N_{n} / n$. Therefore,

$$
\sqrt{n}\left\|\mathbb{F}_{n}^{*}-\tilde{\mathbb{F}}_{n}\right\|=\frac{N_{n}}{\sqrt{n}} \sup _{t \in[0, K]}\left(1-\mathbb{F}_{n}^{*}(t)\right) \leq \frac{N_{n}}{\sqrt{n}} \stackrel{\mathrm{P}}{\rightarrow} 0
$$

by assumption.

Regarding the second term of the right-hand side of (3), notice that $\tilde{Y}_{i} \leq Y_{i} \leq \tilde{Y}_{i}+\epsilon_{n}$, where $\epsilon_{n}=d_{H}\left(\partial S, \partial S_{n}\right)$. Then,

$$
\sqrt{n}\left\|\tilde{\mathbb{F}}_{n}-\mathbb{F}_{n}\right\| \leq \sup _{t \in[0, K]} \frac{1}{\sqrt{n}} \sum_{i=1}^{n} \mathbb{I}_{\left\{t<Y_{i} \leq t+\epsilon_{n}\right\}}
$$

Define the sequence $b_{n}=n^{-1 / 2-\delta}$, where $\delta>0$ is given in the assumptions of the lemma. Notice that, from the assumption on $d_{H}\left(\partial S, \partial S_{n}\right)$, we have $b_{n}>\epsilon_{n}$ eventually with probability 1 . Then,

$$
\sup _{t \in[0, K]} \frac{1}{\sqrt{n}} \sum_{i=1}^{n} \mathbb{I}_{\left\{t<Y_{i} \leq t+\epsilon_{n}\right\}} \leq \sup _{t \in[0, K]} \frac{1}{\sqrt{n}} \sum_{i=1}^{n} \mathbb{I}_{\left\{t<Y_{i} \leq t+b_{n}\right\}}, \text { eventually with probability } 1 .
$$

Now, denote by $C_{n}$, for each $n$, the covering of $(0, K]$ by intervals of the form $I_{j}^{n}=\left(j b_{n},(j+\right.$ 1) $\left.b_{n}\right], j=1,2, \ldots$. Clearly, the cardinality of $C_{n}$ is $O\left(b_{n}^{-1}\right)=O\left(n^{\gamma}\right)$ with $\gamma=1 / 2+\delta$. Also, since $F$, the distribution of the $Y_{i}$ 's, is Lipschitz continuous, there exists $M$ such that

$$
\max _{I \in C_{n}} \mathbb{P}_{F}(I) \leq M b_{n}=o\left(n^{-1 / 2}\right) .
$$

Therefore, the sequence of coverings $C_{n}$ fulfills the assumptions in Lemma 2.2 of Fernholz (1991). It follows that $T_{n} / \sqrt{n} \stackrel{\text { a.s. }}{\rightarrow} 0$, where $T_{n}$ is the maximum number of $Y_{i}$ 's with values in any $I \in C_{n}$. Then,

$$
\sup _{t \in[0, K]} \frac{1}{\sqrt{n}} \sum_{i=1}^{n} \mathbb{I}_{\left\{t<Y_{i} \leq t+b_{n}\right\}} \leq \frac{2 T_{n}}{\sqrt{n}} \stackrel{\text { a.s. }}{\rightarrow} 0
$$

From (4), (5) and (6) we get $\sqrt{n}\left\|\tilde{\mathbb{F}}_{n}-\mathbb{F}_{n}\right\| \stackrel{\text { a.s. }}{\rightarrow} 0$.

Proof of Lemma 3. Applying the triangle inequality,

$$
\left\|\mathbb{F}_{n}^{*}-\hat{F}\right\| \leq\left\|\mathbb{F}_{n}^{*}-\mathbb{F}_{n}\right\|+\left\|\mathbb{F}_{n}-F\right\|+\|F-\hat{F}\|
$$

and

$$
\left\|\mathbb{F}_{n}-F\right\| \leq\left\|\mathbb{F}_{n}-\mathbb{F}_{n}^{*}\right\|+\left\|\mathbb{F}_{n}^{*}-\hat{F}\right\|+\|\hat{F}-F\|
$$

Hence,

$$
D_{n}-\sqrt{n}\left\|\mathbb{F}_{n}^{*}-\mathbb{F}_{n}\right\|-\sqrt{n}\|F-\hat{F}\| \leq D_{n}^{*} \leq D_{n}+\sqrt{n}\left\|\mathbb{F}_{n}^{*}-\mathbb{F}_{n}\right\|+\sqrt{n}\|F-\hat{F}\|
$$

and the result follows from Lemmas 1 and 2. 
Proof of Theorem 1. We are going to check the assumptions of Lemma 3. By Theorem 3 and Remark 3 in Rodríguez-Casal (2007), it holds

$$
d_{H}\left(\partial S, \partial S_{n}\right)=O\left(\frac{\log n}{n}\right)^{2 / 3}
$$

with probability 1 . Observe that, if $S$ is convex and $S_{n}, C_{\lambda}\left(\mathcal{X}_{n}\right)$ stand for the convex hull and the $\lambda$-convex hull, respectively, of $\mathcal{X}_{n}$, then $C_{\lambda}\left(\mathcal{X}_{n}\right) \subset S_{n} \subset S$ for any $\lambda>0$. Thus, $n^{1 / 2+\delta} d_{H}\left(\partial S, \partial S_{n}\right) \stackrel{\text { ass }}{\rightarrow} 0$, for $0<\delta<1 / 6$.

Theorem 1 in Schütt (1994) ensures that for any convex body $S \subset \mathbb{R}^{d}, \mathbb{E}\left[\mu\left(S \backslash S_{n}\right)\right]=$ $O\left(n^{-2 /(d+1)}\right)$. In particular, for $d=2$ and using Markov inequality we have $n^{\beta} \mu\left(S \backslash S_{n}\right) \stackrel{\text { P }}{\rightarrow} 0$, for $0 \leq \beta<2 / 3$.

Finally, we use the so-called Efron's identity, see Efron (1965, Eq. 3.7), which relates the expected number of vertices and the area in the convex hull $S_{n}$, and we get

$$
\mathbb{E}\left(N_{n}\right)=n \frac{\mathbb{E}\left[\mu\left(S \backslash S_{n-1}\right)\right]}{\mu(S)}=O\left(n^{(d-1) /(d+1)}\right),
$$

where in the second equality we have used again Schütt's Theorem. In particular, for $d=2$ and using Markov's inequality we have $N_{n} / n^{\beta} \stackrel{\text { P }}{\rightarrow} 0$, for $\beta>1 / 3$.

Proof of Theorem 2. We are going to check the assumptions of Lemma 3. By Theorem 3 in Rodríguez-Casal (2007), with probability 1

$$
d_{H}\left(\partial S, \partial S_{n}\right)=O\left(\frac{\log n}{n}\right)^{2 / 3}
$$

and the same rate holds for $\mu\left(S \backslash S_{n}\right)$. Then, $n^{\beta} \mu\left(S \backslash S_{n}\right) \stackrel{\text { a.s. }}{\rightarrow} 0$, for $0 \leq \beta<2 / 3$, and $n^{1 / 2+\delta} d_{H}\left(\partial S, \partial S_{n}\right) \stackrel{\text { a.s. }}{\rightarrow} 0$, for $0<\delta<1 / 6$. Finally we have $\mathbb{E}\left(N_{n}\right)=O\left(n^{1 / 3}\right)$ (see PateiroLópez \& Rodríguez-Casal, 2011) and using Markov inequality we obtain $N_{n} / n^{\beta} \mathrm{p} \rightarrow 0$ for $\beta>1 / 3$.

\section{ACKNOWLEDGEMENTS}

This work was partially supported by grants from the Spanish Ministry of Science and Innovation, and Comunidad de Madrid (first and second author) and by grants from the Spanish Ministry of Science and Innovation, and the Belgian government through the IAP research network (third author).

\section{BIBLIOGRAPHY}

Berrendero, J. R., Cuevas, A., \& Vázquez-Grande, F. (2006). Testing multivariate uniformity: The distanceto-boundary method. The Canadian Journal of Statistics, 34(4), 693-707.

Berrendero, J.R., Cuevas, A., \& Pateiro-López, B. (2011). A multivariate uniformity test for the case of unknown support. Statistics and Computing, http://dx.doi.org/10.1007/s11222-010-9222-z.

Cuevas, A. \& Fraiman, R. (2009). Set estimation. In Kendall W. S. \& Molchanov, I. (Eds.) New Perspectives on Stochastic Geometry, Oxford University Press, 374-397.

Devroye, L. (1986). Nonuniform random variate generation, Springer-Verlag, New York.

DOI: 
Dümbgen, L. \& Walther, G. (1996). Rates of convergence for random approximation of convex sets. Advances in Applied Probability, 28(2), 384-386.

Fernholz, L.T. (1991). Almost sure convergence of smoothed empirical distribution functions. Scandinavian Journal of Statistics, 18(3), 255-262.

Edelsbrunner, H. \& Mücke, E. P. (1994). Three-dimensional Alpha Shapes. ACM Transactions on Graphics, 13(1), 43-72.

Efron, B. (1965). The convex hull of a random set of points. Biometrika, 52(3/4), 331-343.

Grasman, R. \& Gramacy, R. B. (2010). geometry: Mesh generation and surface tesselation. R package version 0.1-7. http://CRAN.R-project.org/package=geometry.

Jain, A., Xu, X., Ho, T., \& Xiao, F. (2002). Uniformity testing using minimal spanning tree. Proceedings of the 16th International Conference on Pattern Recognition, 4, 281-284.

Jaklič, A., Leonardis, A., \& Solina, F. (2000). Segmentation and Recovery of Superquadrics: Computational imaging and vision, Kluwer.

Janson, S. (1987). Maximal spacings in several dimensions. The Annals of Probability, 15(1), 274-280.

Liang, J. J., Fang, K. T., Hickernell, F. J., \& Li, R. (2001). Testing multivariate uniformity and its applications. Mathematics of Computation, 70(233), 337-355.

Pateiro-López, B. \& Rodríguez-Casal, A. (2010). Generalizing the convex hull of a sample: The R package alphahull. Journal of Statistical Software, 34(5), 1-28.

Pateiro-López, B. \& Rodríguez-Casal, A. (2011). Recovering the shape of a point cloud in the plane. arXiv:1105.5945v1.

Pegden, W. (2011). Sets resilient to erosion. Advances in Geometry, 11(2), 201-224.

Perkal, J. (1956). Sur les ensembles $\epsilon$-convexes. Colloquium Mathematicae, 4, 1-10.

R Development Core Team. (2011). R: A Language and Environment for Statistical Computing. R Foundation for Statistical Computing, Vienna, Austria. http://www.R-project.org.

Reitzner, M. (2009). Random polytopes. In Kendall W. S. \& Molchanov, I. (Eds.) New Perspectives on Stochastic Geometry, Oxford University Press, 45-76.

Rodríguez-Casal, A. (2007). Set estimation under convexity type assumptions. Annales de l'Institut Henri Poincare (B) Probability and Statistics, 43(6), 763-774.

Rosin, P.L. \& West, G. A. W. (1995). Curve Segmentation and Representation by Superellipses. IEE Proceedings Vision, Image and Signal Processing, 142(5), 280-288.

Schütt, C. (1994). Random polytopes and affine surface area. Mathematische Nachrichten, 170(1), 227-249.

Walther, G. (1997). Granulometric smoothing. The Annals of Statistics, 25(6), 2273-2299.

Walther, G. (1999). On a generalization of Blaschke's rolling theorem and the smoothing. Mathematical Methods in the Applied Sciences, 22(4), 301-316.

Received 9 July 2009

Accepted 8 July 2010 